\title{
On non-intersecting arithmetic progressions
}

by

\section{Ernest S. Croot III (Berkeley, CA)}

I. Introduction. Suppose that $a_{1}\left(\bmod q_{1}\right), a_{2}\left(\bmod q_{2}\right), \ldots, a_{k}\left(\bmod q_{k}\right)$ is a collection of arithmetic progressions, where $2 \leq q_{1}<\ldots<q_{k} \leq x$, with the property that

$$
\left\{a_{i}\left(\bmod q_{i}\right)\right\} \cap\left\{a_{j}\left(\bmod q_{j}\right)\right\}=\emptyset \quad \text { if } i \neq j .
$$

We say that such a collection of arithmetic progressions is disjoint or nonintersecting. Let $f(x)$ be the maximum value for $k$, maximized over all choices of progressions $a_{i}\left(\bmod q_{i}\right)$. Define

$$
\begin{aligned}
L(c, x) & :=\exp (c \sqrt{\log x \log \log x}), \\
\psi(x, y) & :=\#\{n \leq y: p \text { prime, } p \mid n \Rightarrow p \leq y\}, \\
\psi^{*}(x, y) & :=\#\left\{n \leq y: p \text { prime, } p^{a} \mid n \Rightarrow p^{a} \leq y\right\} .
\end{aligned}
$$

In [3], Erdős and Szemerédi prove that

$$
\frac{x}{\exp \left((\log x)^{1 / 2+\varepsilon}\right)}<f(x)<\frac{x}{(\log x)^{c}}
$$

for some constant $c>0$. (This result is also mentioned in [2].) Their lower bound can be refined by using more exact estimates for $\psi(x, L(c, x))$ than was used in their paper. Specifically, as direct consequence of [1, Lemma 3.1], we have the following estimate:

Lemma 1. For any constant $c>0$,

$$
\psi(x, L(c, x))=\frac{x}{L(1 /(2 c)+o(1), x)} .
$$

We also have the same estimate for $\psi^{*}(x, L(c, x))$, since

$$
\begin{aligned}
\psi(x, L(c, x)) & >\psi^{*}(x, L(c, x)) \\
& >\psi(x, L(c, x))-\sum_{n^{2}>L(c, x)} \psi\left(x / n^{2}, L(c, x)\right)
\end{aligned}
$$

2000 Mathematics Subject Classification: Primary 11B25. 


$$
=\psi(x, L(c, x))-O\left(\frac{x}{L(c / 2+1 /(2 c)+o(1), x)}\right) .
$$

Now, let $p$ be the largest prime number less than or equal to $L(1 / \sqrt{2}, x)$. Let $q_{1}, \ldots, q_{t}$ be the collection of all integers $\leq x$ which are divisible by $p$, and whose prime power factors are all $<p$. From (1) and (2), we deduce that

$$
t=\frac{x}{p L(1 / \sqrt{2}+o(1), x)}=\frac{x}{L(\sqrt{2}+o(1), x)} .
$$

For each $q_{i}=p l_{r}^{h_{r}} l_{r-1}^{h_{r-1}} \ldots l_{1}^{h_{1}}$, where $p>l_{r}^{h_{r}}>l_{r-1}^{h_{r-1}}>\ldots>l_{1}^{h_{1}}$ are the powers of the distinct primes dividing $q_{i}$, we choose the residue class $a_{i}$ $\left(\bmod q_{i}\right)$ using the Chinese Remainder Theorem as follows:

$$
a_{i} \equiv l_{r}^{h_{r}}(\bmod p) ; \quad a_{i} \equiv l_{j-1}^{h_{j-1}}\left(\bmod l_{j}^{h_{j}}\right) \quad \text { for } 2 \leq j \leq r
$$

and finally,

$$
a_{i} \equiv 0\left(\bmod l_{1}^{h_{1}}\right) .
$$

This is exactly the construction which appears in [3] (except that their progressions were all square-free), and it is easy to see that our progressions $a_{i}\left(\bmod q_{i}\right)$ are disjoint. Thus, we have

$$
f(x)>\frac{x}{L(\sqrt{2}+o(1), x)} .
$$

In this paper we will prove the following results:

THEOREM 1. If $a_{1}\left(\bmod q_{1}\right), \ldots, a_{k}\left(\bmod q_{k}\right)$ are a collection of disjoint arithmetic progressions, where the $q_{i}$ 's are square-free and $2 \leq q_{1}<\ldots<$ $q_{k} \leq x$, then

$$
k<\frac{x}{L(1 / 2-o(1), x)} .
$$

Corollary to Theorem 1.

$$
f(x)<\frac{x}{L(1 / 6-o(1), x)} .
$$

Thus, we will have shown that

$$
\frac{x}{L(\sqrt{2}+o(1), x)}<f(x)<\frac{x}{L(1 / 6-o(1), x)} .
$$

To see how the Corollary follows from Theorem 1 , let $b_{1}\left(\bmod r_{1}\right), \ldots, b_{f(x)}$ $\left(\bmod r_{f(x)}\right)$ be a maximal collection of disjoint arithmetic progressions with $2 \leq r_{1}<\ldots<r_{f(x)} \leq x$. Suppose, for proof by contradiction, that for some $\varepsilon<1 / 6$

$$
f(x)>\frac{x}{L(1 / 6-\varepsilon, x)} .
$$

Write each $r_{i}=\alpha_{i} \beta_{i}$, where $\beta_{i}$ is square-free, $\operatorname{gcd}\left(\alpha_{i}, \beta_{i}\right)=1$, and every prime dividing $\alpha_{i}$ divides to a power $\geq 2$. (Note: we may have $\alpha_{i}$ or $\beta_{i}=1$.) 
Now, at least half of $\alpha_{i}$ 's must be $\leq L(1 / 3, x)$, for if not we would deduce from our assumption (3) that

$$
\begin{aligned}
\frac{x}{2 L(1 / 6-\varepsilon, x)} & <f(x) / 2<\#\left\{r_{i}: \alpha_{i}>L(1 / 3, x)\right\} \\
& <x \sum_{n^{2}>L(1 / 3, x)} \frac{1}{n^{2}} \prod_{p \text { prime }}\left(1+\frac{1}{p^{2}}+\frac{1}{p^{3}}+\ldots\right) \\
& \ll \frac{x}{L(1 / 6, x)},
\end{aligned}
$$

which is impossible for $x$ large enough in terms of $\varepsilon$. Thus, there must exist an $\alpha<L(1 / 3, x)$ for which at least $f(x) /(2 L(1 / 3, x))$ of the $r_{i}$ 's have $\alpha_{i}=\alpha$. Let $R(\alpha) \subseteq\left\{r_{1}, \ldots, r_{f(x)}\right\}$ be such a collection of $r_{i}$ 's, where

$$
|R(\alpha)|>\frac{f(x)}{2 L(1 / 3, x)}>\frac{x}{2 L(1 / 2-\varepsilon, x)} ;
$$

this last inequality follows from our assumption (3). Now there must exist a residue class $b(\bmod \alpha)$ for which at least $|R(\alpha)| / \alpha$ of the progressions $b_{i}$ $\left(\bmod r_{i}\right)$ satisfy

$$
r_{i} \in R(\alpha) \quad \text { and } \quad b_{i} \equiv b(\bmod \alpha) .
$$

Thus, the arithmetic progressions $b_{i}\left(\bmod r_{i} / \alpha\right)$, where $r_{i}$ satisfies (4), form a collection of $\geq|R(\alpha)| / \alpha \gg x /(\alpha L(1 / 2-\varepsilon, x))$ disjoint progressions, with distinct square-free moduli $\leq x / \alpha$. This contradicts Theorem 1 for $x$ sufficiently large in terms of $\varepsilon$. We must conclude, therefore, that the bound in (3) is false for all $\varepsilon<1 / 6$ and $x>x_{0}(\varepsilon)$, and so the Corollary to Theorem 1 follows.

II. Proof of Theorem 1. Before we prove Theorem 1, we will need the following lemma:

Lemma 2. There are at most $x / L(c / 2+o(1), x)$ positive integers $n \leq x$ such that $\omega(n)>c \sqrt{\log x / \log \log x}\left(\right.$ recall: $\left.\omega(n)=\sum_{p \mid n, p \text { prime }} 1\right)$, where $c$ is some positive constant.

Proof. We observe that

$$
\begin{gathered}
\#\{n \leq x: \omega(n)>c \sqrt{\log x / \log \log x}\}<x \sum_{j>c \sqrt{\frac{\log x}{\log \log x}} \frac{\left(\sum_{p^{a} \leq x, p \text { prime }} 1 / p^{a}\right)^{j}}{j !}} \\
=\frac{x}{(c \sqrt{\log x / \log \log x})^{\{c+o(1)\} \sqrt{\log x / \log \log x}}} \\
=\frac{x}{L(c / 2+o(1), x)} .
\end{gathered}
$$


We now resume the proof of Theorem 1 . Consider the collection of all the $q_{i}$ 's with the properties:

(A) $\omega\left(q_{i}\right)<\sqrt{\log x / \log \log x}$.

(B) There exists a prime $p>L(1, x)$ such that $p \mid q_{i}$.

Let $\left\{r_{1}, \ldots, r_{k^{\prime}}\right\}$ be the collection of all $q_{i}$ 's satisfying $(\mathrm{A})$ and $(\mathrm{B})$, and let $\left\{b\left(r_{1}\right), \ldots, b\left(r_{k^{\prime}}\right)\right\}$ be their corresponding residue classes.

To prove our theorem, we start with the set $S_{0}=\left\{r_{1}, \ldots, r_{k^{\prime}}\right\}$, and construct a sequence of subsets $S_{0} \supseteq S_{1} \supseteq S_{2} \supseteq \ldots$, and a sequence of primes $p_{1}, p_{2}, \ldots$ (and let $p_{0}=1$ ), such that for each $i \geq 1$, the following three properties hold:

1. Each member of $S_{i}$ is divisible by the primes $p_{1}, \ldots, p_{i}$.

2. There exists an integer $A_{i}$ such that for each $r_{j} \in S_{i}$, we have $b\left(r_{j}\right) \equiv A_{i}$ $\left(\bmod p_{1} \ldots p_{i}\right)$.

3. $\left|S_{i}\right|>\left|S_{i-1}\right| /\left(p_{i} \sqrt{\log x / \log \log x}\right)$.

We continue constructing these subsets until we reach a subset $S_{t}$ which has the additional property:

4. There exists a prime $p \neq p_{1}, \ldots, p_{t}, p \geq L(1, x)$, such that at least $\left|S_{t}\right| / \sqrt{\log x / \log \log x}$ of the elements of $S_{t}$ are divisible by $p$.

Let us suppose for the time being that we can construct these sets $S_{1}, \ldots, S_{t}$. Applying property 3 iteratively, together with property 4 , we find that the number of elements of $S_{t}$ which are divisible by $p$ (which are already divisible by $p_{1} \ldots p_{t}$ by property 1 ) is at least

$$
\frac{\left|S_{0}\right|}{p_{1} \ldots p_{t}(\sqrt{\log x / \log \log x})^{t+1}} \geq \frac{\left|S_{0}\right|}{p_{1} \ldots p_{t} L(1 / 2+o(1), x)} .
$$

(Note: By property (A) above we have $t \leq \sqrt{\log x / \log \log x}$ since every element of $S_{0}$ has at most $\sqrt{\log x / \log \log x}$ prime factors.) From this, together with the fact that $p>L(1, x)$, we have

$$
\begin{aligned}
\frac{x}{p_{1} \ldots p_{k} L(1, x)} & \geq \#\left\{n \leq x: p p_{1} \ldots p_{t} \mid n\right\}>\#\left\{q \in S_{t}: p \mid q\right\} \\
& \geq \frac{\left|S_{0}\right|}{p_{1} \ldots p_{t} L(1 / 2+o(1), x)} .
\end{aligned}
$$

It follows that

$$
\left|S_{0}\right|<\frac{x}{L(1 / 2-o(1), x)}
$$

From this, together with Lemmas 1 and 2 and the fact that the elements of 
$S_{0}$ satisfy (A) and (B) above, we have

$$
\begin{aligned}
& \frac{x}{L(1 / 2-o(1), x)}>\left|S_{0}\right|>k-\#\{n \leq x: \omega(n) \geq \sqrt{\log x / \log \log x}\} \\
&>-\psi(x, L(1, x)) \\
&> \frac{x}{L(1 / 2-o(1), x)}
\end{aligned}
$$

and so

$$
k<\frac{x}{L(1 / 2-o(1), x)},
$$

which proves our theorem.

To construct our sets $S_{i}$, we apply the following iterative procedure: suppose we have constructed the sets $S_{1}, \ldots, S_{i}$ which satisfy 1 through 3 above. To construct $S_{i+1}$, first pick any element $r \in S_{i}$. Now let $e_{1}, \ldots, e_{j}$ be all those primes dividing $r /\left(p_{1} \ldots p_{i}\right)$ (note: $j<\sqrt{\log x / \log \log x}$ ). Each element $s \in S_{i}, s \neq r$, is divisible by at least one of these primes, since otherwise $\operatorname{gcd}(r, s)=p_{1} \ldots p_{i}$ and so we would have $b(r) \equiv A_{i} \equiv b(s)$ $(\bmod \operatorname{gcd}(r, s))$, which would mean that $\{b(r)(\bmod r)\} \cap\{b(s)(\bmod s)\} \neq \emptyset$.

Now, there must be at least $\left|S_{i}\right| / j>\left|S_{i}\right| / \sqrt{\log x / \log \log x}$ of the elements of $S_{i}$ which are divisible by one of these primes $e_{h}$. Let $C_{i} \subseteq S_{i}$ be the collection of all elements $S_{i}$ divisible by this prime $e_{h}$. There exists at least one residue class $B\left(\bmod e_{h}\right)$ for which more than $\left|C_{i}\right| / e_{h}>$ $\left|S_{i}\right| /\left(e_{h} \sqrt{\log x / \log \log x}\right)$ of the elements $r \in C_{i}$ satisfy $b(r) \equiv B\left(\bmod e_{h}\right)$. Now let $S_{i+1}$ be the collection of all such $r \in C_{i}$, set $p_{i+1}=e_{h}$, and let $A_{i+1} \equiv A_{i}\left(\bmod p_{1} \ldots p_{i}\right)$ and $A_{i+1} \equiv B\left(\bmod p_{i+1}\right)$ by the Chinese Remainder Theorem. Then properties 1,2 , and 3 above follow immediately for this set $S_{i+1}$.

If there exists a prime $p>L(1, x)$ which divides more than

$$
\frac{\left|S_{i+1}\right|}{\sqrt{\log x / \log \log x}}
$$

of the elements of $S_{i+1}$, then we set $t=i+1$ and we are finished. If not, we continue constructing these sets $S_{j}$. We are guaranteed to eventually hit upon such a prime $p$ since all our $r_{j}$ 's are divisible by at least one prime $p>L(1, x)$ by property (B).

\section{References}

[1] E. R. Canfield, P. Erdős, and C. Pomerance, On a problem of Oppenheim concerning "factorisatio numerorum", J. Number Theory 17 (1983), 1-28.

[2] P. Erdős and R. L. Graham, Old and New Problems and Results in Combinatorial Number Theory, Monograph. Enseign. Math. 28, Univ. Genève, 1980. 
[3] P. Erdős and E. Szemerédi, On a problem of P. Erdös and S. Stein, Acta Arith. 15 (1968), 85-90.

Department of Mathematics

U. C. Berkeley

1067 Evans Hall

Berkeley, CA 94720, U.S.A.

E-mail: ecroot@math.berkeley.edu 